\title{
Interdiffusion Data in Multicomponent Alloys as a Source of Quantitative Fundamental Diffusion Information
}

\author{
I.V. Belova ${ }^{1, a}$ and G.E. Murch ${ }^{1, b}$ \\ ${ }^{1}$ Diffusion in Solids Group, School of Engineering, \\ The University of Newcastle, Callaghan, NSW 2308, Australia \\ arina.Belova@newcastle.edu.au, bGraeme.Murch@newcastle.edu.au
}

Keywords: Diffusion, interdiffusion, chemical diffusion, intrinsic diffusion, tracer correlation.

\begin{abstract}
Tracer diffusion experiments have historically furnished much of the information about fundamental diffusion processes as embodied in such quantities as tracer correlation factors and vacancy-atom exchange frequencies. As tracer diffusion experiments using radiotracers are rather less often performed nowadays, it is important to be able to process other diffusion data to provide similar fundamental information. New procedures that are primarily based around the random alloy model have been established recently for analyzing chemical diffusion data in binary and ternary alloy systems. These procedures are reviewed here. First, we review the random alloy model, the Sum-rule relating the phenomenological coefficients and three diffusion kinetics formalisms making use of the random alloy. Next, we show how atom-vacancy exchange frequency ratios and then component tracer correlation factors can be extracted from chemical diffusion data in alloy systems. Examples are taken from intrinsic diffusion and interdiffusion data in a number of binary and ternary alloys.
\end{abstract}

\section{Introduction}

It is interesting to note that it was a chemical diffusion experiment performed almost sixty years ago showing the Kirkendall shift that provided the definitive evidence to the diffusion community that vacancies are the principal vehicles by which atoms migrate in metals and alloys [1]. Over the years, however, tracer diffusion experiments, by way of isotope effect measurements [2], analysis of curvatures of Arrhenius plots [3], pressure dependence [4], analysis of enhancement factors for solvent diffusion in dilute alloys [5], Haven Ratio measurements (in ionic conductors) [6], composition dependence [7], have provided almost all of the fundamental information about atomic diffusion processes. For a variety of reasons however, relatively few laboratories world-wide continue to perform measurements of tracer diffusion coefficients using radioisotopes though it needs to be acknowledged that this trend has been offset to some extent by the widespread use of SIMS measurements using stable isotopes.

Chemical or interdiffusion diffusion data are generally obtained for the very practical reasons of assisting the fabrication/synthesis of engineering materials or predicting the extent of reaction with adjoining metals in-service. Such data have very rarely been analyzed to provide fundamental information about diffusion, by which we mean atom-vacancy exchange frequencies and correlations between jump directions of the atoms as embodied in correlation factors. The amount of chemical/interdiffusion diffusion data is very large; see, for example, the extensive compilations for interdiffusion data in binary alloys [8] and ternary alloys [9]. In view of the above, it is appropriate therefore to seek and develop new ways of extracting fundamental diffusion information from such data. Nonetheless, it needs to be recognized that the accuracy of chemical diffusion data does not usually approach what has been routinely possible in tracer diffusion experiments performed on metals and alloys [10]. There are several reasons for this. Tracer diffusion experiments are normally carried out on well-annealed specimens where the vacancy concentration is at equilibrium. Whilst the starting materials of an interdiffusion couple will generally be well-annealed, during the interdiffusion experiment itself, vacancies are created and annihilated at sources and sinks, typically by dislocation climb. (This creation/annihilation of 
vacancies occurs because the diffusion coefficients of the components themselves are normally unequal which leads to a net vacancy flux, and the Kirkendall effect.) If these vacancy sources and sinks are not efficient or are not at sufficiently high densities, then vacancy super-saturation and under-saturation during interdiffusion can readily occur. It is not easy to estimate the effects on interdiffusion of such possible non-equilibrium vacancy concentrations [11]. Furthermore, in contrast to standard tracer serial sectioning experiments, it is also not straightforward to estimate possible contributions to chemical diffusion processes from grain boundary diffusion processes. Indeed, it is probably fair to say that rather little is known about chemical diffusion in the presence of grain boundaries.

In this paper, we review some of the recent methods in the analysis of chemical diffusion data in order to extract fundamental diffusion information. In the next section we introduce the random alloy model: this forms the basis of the analysis of chemical diffusion in binary and ternary alloys. In that section we also introduce the Sum-rule relationships between the phenomenological transport coefficients, again for the random alloy. In the following section we introduce three diffusion kinetics formalisms that can be used with the random alloy model. In the following section we show how the Sum-rule and these diffusion kinetics formalisms can be used to extract exchange frequency ratios and tracer correlation factors from intrinsic diffusion coefficients that have been measured in binary and ternary alloy systems. In the final section we focus on the analysis of interdiffusion coefficients in binary and ternary systems and show how exchange frequency ratios and tracer correlation factors quantities can be extracted from interdiffusion data.

\section{The Random Alloy Model and the Sum-Rule}

The Random Alloy Model. In the random alloy model introduced first by Manning [12] the $N$ atomic components and independent vacancies (at a vanishingly small concentration) are distributed randomly. The atom-vacancy exchange frequencies, notated as $w_{\mathrm{i}}$ can be conceptualized in one of two ways. On the one hand, one can consider them simply as explicit frequencies that depend only on the species of atom and not on the surroundings. For example $w_{\mathrm{A}}$ simply represents the basic vacancy- atom frequency of a given $A$ atom at all compositions. On the other hand, one can also consider $w_{\mathrm{A}}$ as representing an average frequency at a given composition. Then $w_{\mathrm{A}}$ represents the average frequency of a given $A$ atom as it migrates through the lattice sampling the various environments. Since the average environment must change with composition, then $w_{\mathrm{A}}$ can also be expected to change with composition to reflect this [13].

The Sum-rule between the Phenomenological Coefficients. A particularly useful and exact result of the random alloy model is the so-called 'Sum-rule' [14] that relates the phenomenological coefficients of non-equilibrium thermodynamics $L_{\mathrm{ij}}$ to the vacancy-atom exchange frequencies and composition. The Sum-rule reduces the number of independent phenomenological coefficients. For example, in the binary alloy, there is only one independent phenomenological coefficient and not three. In the ternary alloy, there are three independent phenomenological coefficients and not six. In the $N$-component alloy the Sum-rule is written as:

$$
\sum_{j=1}^{N} \frac{L_{i j}}{c_{i}} \frac{w_{i}}{w_{j}}=A w_{i}, \quad i=1, \ldots, N
$$

where $A$ is a constant. In terms of the collective correlation factors, which are the correlated parts of the phenomenological coefficients [15], Eq. 1 is rewritten as: 
$\sum_{j=1}^{N} \frac{f_{i j}^{(j)}}{w_{i}} w_{j}=1, \quad$ all $j$

where $f_{i j}^{(j)}$ are the collective correlation factors, sometimes also called correlation functions. Some examples of the immediate usefulness of the Sum-rule in chemical diffusion problems are provided in the next section.

\section{Diffusion Kinetics Formalisms for use with the Random Alloy}

The Darken Theory. Although not originally intended as diffusion kinetics formalism for the random alloy, it is convenient nonetheless to treat the Darken theory [16] here along with the other formalisms. There are various manifestations of the Darken theory depending on the diffusion context. The common feature however is that all off-diagonal phenomenological coefficients are first put equal to zero. This means that all correlation information as embodied in tracer correlation factors, collective correlation factors and vacancy-wind factors is ignored. The principal result of this is that the atoms of all species are assumed to always follow uncorrelated random walks thereby implying that all of the tracer correlation factors are simply given by:

$$
f_{\mathrm{i}} \equiv 1
$$

Similarly, all vacancy-wind factors appearing in expressions between tracer diffusion coefficients and interdiffusion and intrinsic diffusion coefficients vanish. Using the Darken approach a very simple relation then exists between the diagonal phenomenological coefficients and the tracer diffusion coefficients:

$$
L_{i i}=c_{i} D_{i}^{*} / k T, \quad L_{i j}=0 \quad \text { for } i \neq j .
$$

The Darken theory trivially follows the Sum-rule given above in Eq. 1.

The Manning Theory. In the Manning diffusion kinetics theory [12], tracer correlation factors of the alloy components are given by the following expression:

$$
f_{i}=\frac{H}{2 \gamma_{i}+H}
$$

with the function $H$ being the positive root of the following equation:

$$
\sum_{i} \frac{\gamma_{i} c_{i}}{2 \gamma_{i}+H}=\frac{1-f_{0}}{2}
$$

where $f_{0}$ is the geometric correlation factor defined by the crystal structure; for example, $f_{0}=$ 0.78145 for the f.c.c. lattice. In Manning's approach, the phenomenological coefficients are directly related to the tracer diffusion coefficients by:

$$
L_{i i}=\frac{c_{i} D_{i}^{*}}{k T}\left(1+\frac{2 c_{i} D_{i}^{*}}{M_{0} \sum_{k} c_{k} D_{k}^{*}}\right), L_{i j}=\frac{2 c_{i} D_{i}^{*} c_{j} D_{j}^{*}}{k T\left(M_{0} \sum_{k} c_{k} D_{k}^{*}\right)}, \text { for } i \neq j .
$$


where $\mathrm{k}$ and $\mathrm{T}$ have their usual meanings and $M_{0}=2 f_{0} /\left(1-f_{0}\right)$ These relations are frequently referred to as the 'Manning Relations'. It is worth noting that the Manning Relations can also be obtained on the basis of two somewhat intuitive assumptions without recourse to the random alloy model itself [17]. These Relations have been shown to be good approximations, even for alloys showing long range order [18].

The Moleko, Allnatt and Allnatt (MAA) Theory. It has been shown by Belova and Murch $[19,20]$ that the self-consistent Moleko, Allnatt and Allnatt (MAA) [21] theory for diffusion kinetics in the random alloy provides the best agreement with results from Monte Carlo simulations for both tracer and collective correlation factors. However, the MAA kinetics equations are a good deal more complicated in structure than those of Manning and in practice considerably more difficult to implement for analysing experimental diffusion data. The expression for the tracer correlation factor $f_{i}$ for the atomic species $i$ is formally similar to that given above in the Manning approach:

$$
f_{i}=\frac{H_{i}}{2 \gamma_{i}+H_{i}}
$$

However, now the functions $H_{i}$ are, in general, different for each species. The tracer correlation factors can be calculated using a system of equations; see, for example, [22]. Using the MAA theory there are no closed-form relations between the phenomenological coefficients and the tracer diffusion coefficients that are equivalent to the Manning Relations (Eq. 7). Nonetheless, it is still possible to use straightforward numerical methods to find all of the $L_{i j}$ from a given set of tracer diffusion coefficients for all atomic species [22].

\section{Analysis of Intrinsic Diffusion Coefficients}

In this section we analyze intrinsic diffusion coefficients by making use of the Sum-rule introduced in Section 2 followed by processing using the Manning and MAA diffusion kinetics formalisms.

Binary Alloy Systems. Intrinsic diffusivities are generally measured by way of marker shifts in the interdiffusion experiment. However, such measurements are rather tedious, especially in ternary alloys and, accordingly, the amount of intrinsic diffusivity data is somewhat limited compared with interdiffusivity data [8,9]. For the random alloy, the Sum-rule provides a surprisingly simple relationship between the ratios of the intrinsic diffusion coefficients and the ratio of the atomvacancy exchange frequencies at a given composition [23]. For example, for a binary alloy, we simply have that:

$$
D_{\mathrm{A}} / D_{\mathrm{B}}=w_{\mathrm{A}} / w_{\mathrm{B}}
$$

where $D_{\mathrm{A}}$ and $D_{\mathrm{B}}$ are the intrinsic diffusivities in the $\mathrm{AB}$ binary alloy and $w_{\mathrm{A}}$ and $w_{\mathrm{B}}$ are the atomvacancy exchange frequencies. Note the absence of any off-diagonal phenomenological coefficients or any type of correlation factor in Eq. 9. Unfortunately, there are no simplifications for the corresponding tracer diffusion coefficients $D^{*}{ }_{\mathrm{A}}$ and $D^{*_{\mathrm{B}}}$ : for the random alloy their ratio simply remains as:

$$
D^{*}{ }_{\mathrm{A}} / D^{*}{ }_{\mathrm{B}}=w_{\mathrm{A}} f_{\mathrm{A}} / w_{\mathrm{B}} f_{\mathrm{B}}
$$

where $f_{\mathrm{A}}$ and $f_{\mathrm{B}}$ are the tracer correlation factors for $\mathrm{A}$ and $\mathrm{B}$. As the first example of the use of Eq. 9, in Fig. 1a we show results for the calculated ratio of the exchange frequencies $w_{N i} / w_{C u}$ directly from the experimental intrinsic diffusivities in the $\mathrm{Cu}-\mathrm{Ni}$ system [24]. As a further example, in Fig. $2 \mathrm{a}$ we show results of the calculated ratio of exchange frequencies $w_{A g} / w_{C d}$ directly from the experimental intrinsic diffusivities in the $A g-C d$ system [25]. In Fig. 2a we also give the ratio of the exchange frequencies as obtained using the ratio of the experimental tracer diffusivities and the Manning formalism. We note that there is quite reasonable agreement of the results of processing these two types of diffusion measurement. 
There has been a very long tradition in the solid-state diffusion research area to determine tracer correlation factors because these factors give very direct information on the degree of correlation in the random walks of the atoms. Tracer correlation factors have frequently been inferred by way of the diffusion isotope effect [2]. (In ionic conductors they can also be inferred from the Haven Ratio [6].) Tracer correlation factors can also be determined from the ratio of the tracer diffusion coefficients (with a knowledge also of the geometric tracer correlation factor $f_{0}$ for the lattice) using the random alloy diffusion kinetics formalisms either of Manning or MAA. Importantly, they can also be determined directly using either of these theories if the ratio of the exchange frequencies is known (together with $f_{0}$ ). In Fig. $1 \mathrm{~b}$ we first show the corresponding tracer correlation factors for $\mathrm{Ni}$ and $\mathrm{Cu}$ as obtained using the Manning formalism from the ratio of the exchange frequencies in Fig. 1a. It is seen that $C u$ atoms have the lower correlation factors and are therefore the most correlated in their motion; this is particularly the case at low $\mathrm{Cu}$ compositions.

(a)

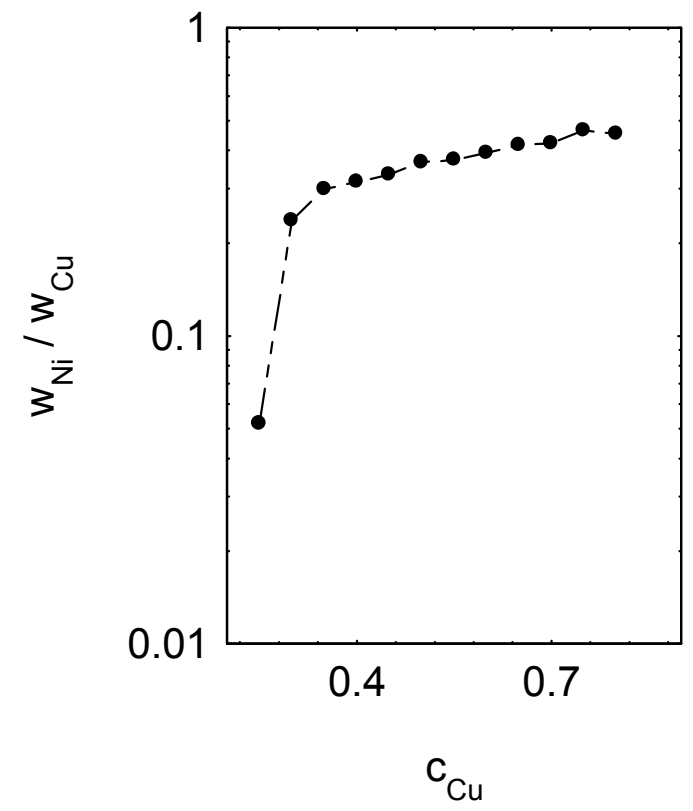

(b)

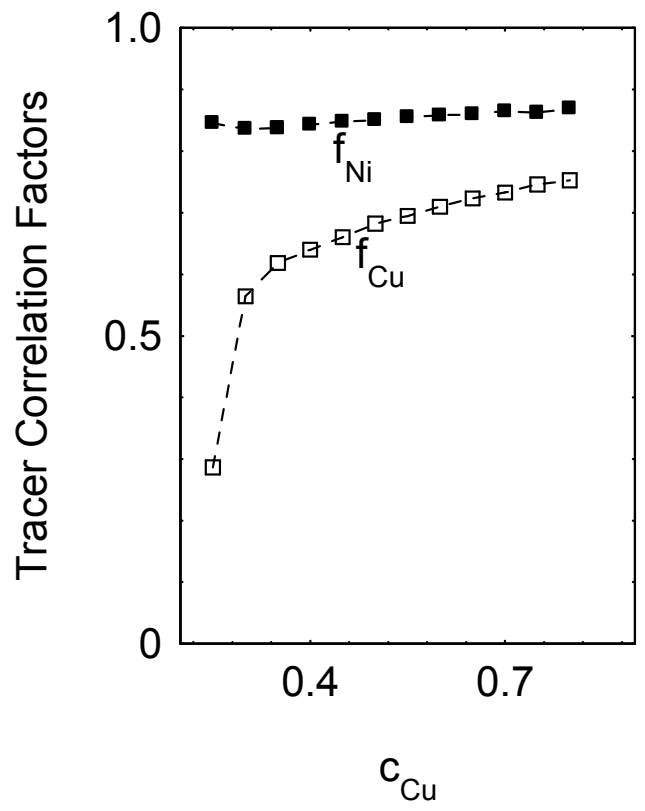

Fig. 1 (a) Ratio of the exchange frequencies for the $\mathrm{Cu}-\mathrm{Ni}$ system at $1273 \mathrm{~K}$ using experimental intrinsic diffusion data [24]. (b) The corresponding tracer correlation factors of $\mathrm{Cu}$ and $\mathrm{Ni}$ after processing the data of (a) using the Manning diffusion kinetics formalism.

Similarly, in Fig. $2 \mathrm{~b}$ we give results for the tracer correlation factors $f_{\mathrm{Ag}}$ and $f_{\mathrm{Cd}}$ determined using the MAA formalism from $w_{A g} / w_{C d}$ and also from the tracer diffusivities [26]. It is seen that $A g$ atoms have the higher tracer correlation factors and therefore have the least correlated motion and vice versa for $C d$ atoms. Again it can be seen that there is quite reasonable agreement for the tracer correlation factors determined from these different diffusion experiments.

Ternary Alloy Systems. For the ternary alloy $A-B-C$ the Sum-rule (Eq. 1) gives the following simple relationship between the exchange frequency ratios and the ternary intrinsic diffusion coefficients: 
(a)

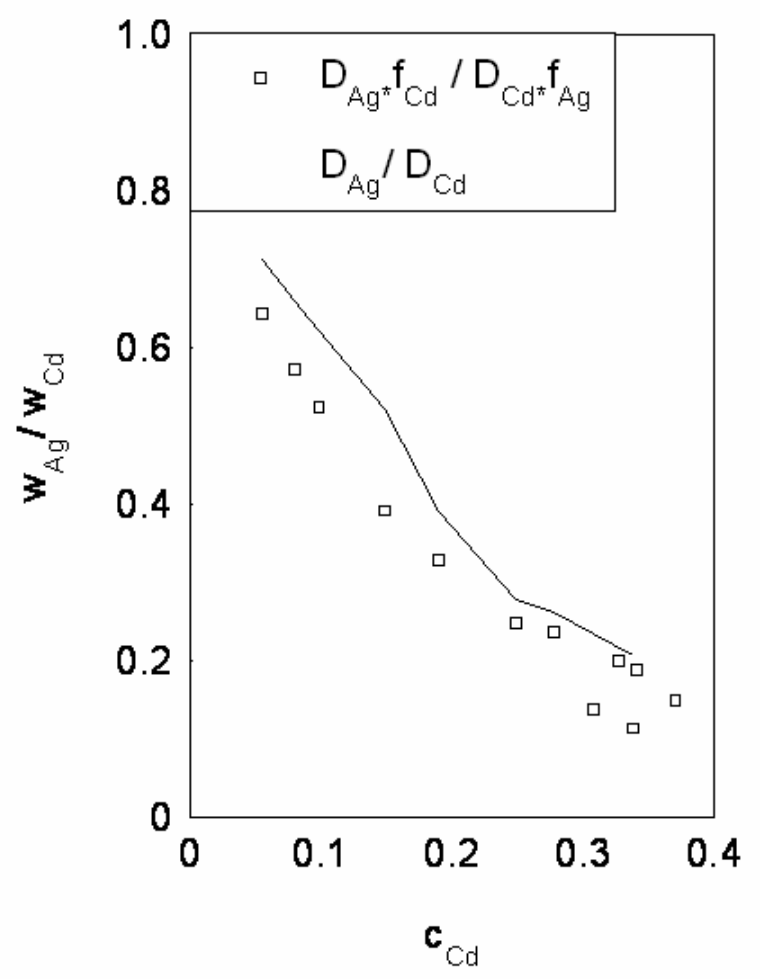

(b)

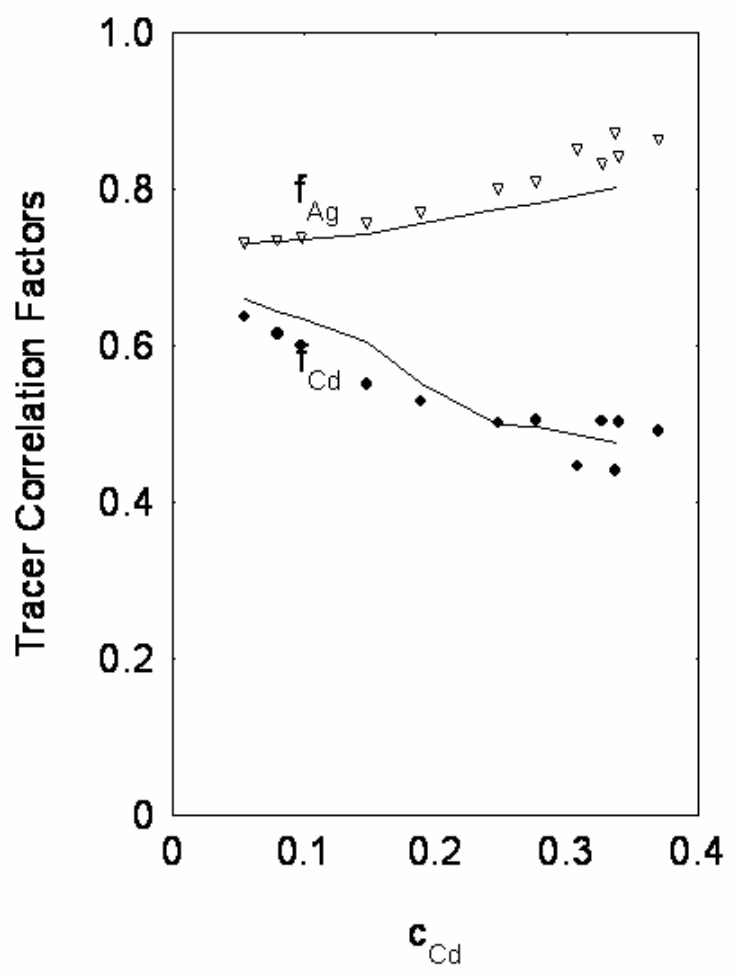

Fig. 2 (a) The ratio $\mathrm{w}_{\mathrm{Ag}} / \mathrm{w}_{\mathrm{Cd}}$ as a function of $\mathrm{c}_{\mathrm{Cd}}$ at $873 \mathrm{~K}$ calculated using intrinsic diffusion coefficients [25] presented as a solid line and calculated from the tracer diffusion coefficients [26] using the Manning formalism and presented as symbols. (b) Corresponding tracer correlation factors, solid lines - calculated using ratio of the intrinsic diffusivities, symbols - calculated using experimental tracer data.

$$
\begin{gathered}
\frac{w_{C}}{w_{A}}=\frac{D_{B A}^{C} D_{C B}^{C}-D_{B B}^{C} D_{C A}^{C}}{D_{A A}^{C} D_{B B}^{C}-D_{A B}^{C} D_{B A}^{C}} \\
\frac{w_{C}}{w_{B}}=\frac{D_{A B}^{C} D_{C A}^{C}-D_{A A}^{C} D_{C B}^{C}}{D_{A A}^{C} D_{B B}^{C}-D_{A B}^{C} D_{B A}^{C}},
\end{gathered}
$$

where $D_{A A}^{C}$ etc are the ternary intrinsic diffusivities in the ternary alloy and $C$ is, by convention the dependent concentration variable. Now we can make use of these equations in the analysis of intrinsic diffusion coefficients in the ternary alloy system $(A g-C d-Z n)$. In Figure 3a we show results for the ratio of the exchange frequencies $w_{\mathrm{Cd}} / w_{\mathrm{Ag}}$ and $w_{\mathrm{Zn}} / w_{\mathrm{Ag}}$ (Eqns. 11 and 12) using intrinsic diffusion data in the $A g-C d-Z n$ system at $\mathrm{T}=873 \mathrm{~K}$ [27]. Again it is possible to gain access to the corresponding tracer correlation factors $f_{\mathrm{Ag}}, f_{\mathrm{Cd}}$ and $f_{\mathrm{Zn}}$. For the ternary alloy system, either the diffusion kinetics formalisms of Manning and MAA can be employed for this task. The results using the MAA formalism are shown in Figure 3b. As in the binary $A g-C d$ system discussed above, it can be seen that the $A g$ atoms are the least correlated in their motion (highest correlation factors). 
(a)

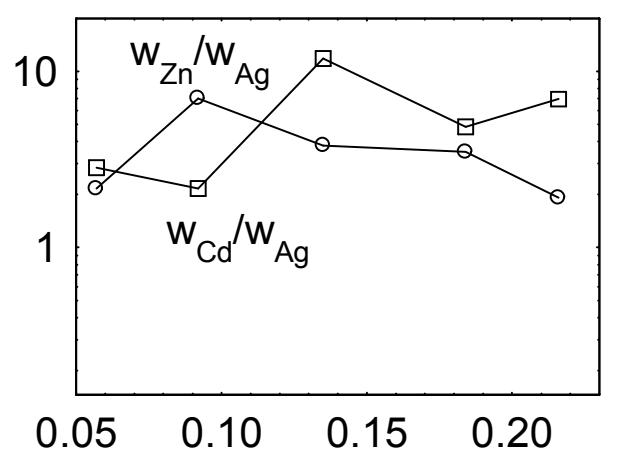

(b)

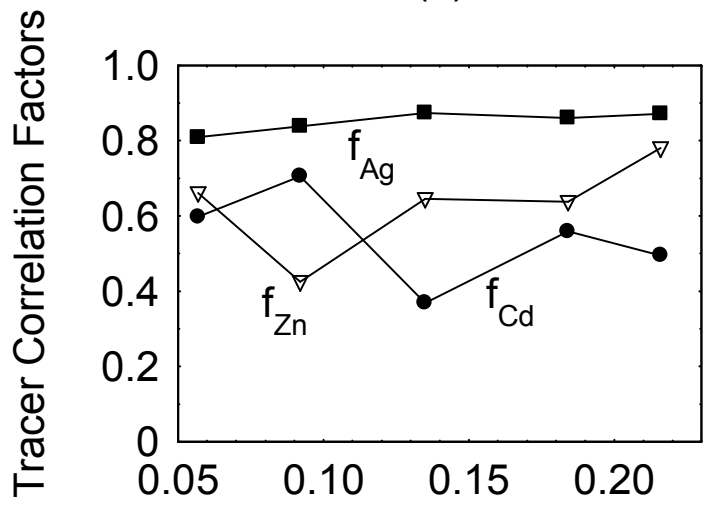

Fig. 3a,b. The ratios of $w_{Z n} / w_{A g}, w_{C d} / w_{A g}$ and the corresponding tracer correlation factors (calculated by making use of the MAA formalism) in $\mathrm{Ag}-\mathrm{Cd}-\mathrm{Zn}$ alloys as a function of composition $\mathrm{c}_{\mathrm{Cd}}, \mathrm{c}_{\mathrm{Zn}}=0.112$.

\section{Analysis of Interdiffusion Coefficients}

Binary Alloys. The processing of the (single) interdiffusion coefficient in a binary alloy cannot be analyzed to give fundamental diffusion information except indirectly by way of its functional form on composition [13]. One possible method involves first expressing the interdiffusion coefficient (after dividing out the thermodynamic factor) in terms of the phenomenological coefficients and then using either Manning or MAA to express the phenomenological coefficients in terms of the ratio of the exchange frequencies. The resulting expression is then used as a fitting parameter to the experimental interdiffusion coefficient (divided by the thermodynamic factor) on composition; the ratio of the exchange frequencies can then be extracted as a fitting parameter. This analysis assumes that the vacancy concentration does not depend on composition (or is known independently) and that the thermodynamic factor is also known independently. The problem with the analysis in practice is that the fitting is numerically very sensitive and requires quite robust data over a very wide composition range to work well.

Ternary alloys. The processing of the four interdiffusivities $\widetilde{D}_{A A}^{C}, \widetilde{D}_{A B}^{C}, \widetilde{D}_{B B}^{C}$ and $\widetilde{D}_{B A}^{C}$ in a ternary alloy system to obtain ratios of exchange frequencies, and then tracer correlation factors, can become quite involved. First, as a zeroth approximation or Darken-type approximation one can simply neglect the off-diagonal phenomenological coefficients in the analysis. It is then straightforward to show that the ratios of the exchange frequencies can be expressed as; see, for example [28]:

$$
\begin{aligned}
& \frac{w_{C}}{w_{A}}=\frac{\left(1-c_{A}\right)\left(\left(1-c_{B}\right)\left(m_{21} \widetilde{D}_{A A}^{C}-m_{11} \widetilde{D}_{A B}^{C}\right)+c_{A}\left(m_{21} \widetilde{D}_{B A}^{C}-m_{11} \widetilde{D}_{B B}^{C}\right)\right)}{c_{A}\left(\left(m_{11}\left(1-c_{B}\right)+m_{12}\right) \widetilde{D}_{A B}^{C}-\left(\left(1-c_{B}\right) m_{21}+m_{22}\right) \widetilde{D}_{A A}^{C}+c_{A} m_{11} \widetilde{D}_{B B}^{C}-c_{A} m_{21} \widetilde{D}_{B A}^{C}\right)} ; \\
& \frac{w_{C}}{w_{B}}=\frac{\left(1-c_{B}\right)\left(m_{21} \widetilde{D}_{A A}^{C}-m_{11} \widetilde{D}_{A B}^{C}\right)+c_{A}\left(m_{21} \widetilde{D}_{B A}^{C}-m_{11} \widetilde{D}_{B B}^{C}\right)}{c_{B} m_{11} \widetilde{D}_{A B}^{C}-c_{B} m_{21} \widetilde{D}_{A A}^{C}-c_{A} m_{11} \widetilde{D}_{B B}^{C}+c_{A} m_{21} \widetilde{D}_{B A}^{C}}
\end{aligned}
$$

or

$$
\frac{w_{C}}{w_{A}}=\frac{\left(1-c_{A}\right)\left(m_{12} \widetilde{D}_{B B}^{C}-m_{22} \widetilde{D}_{B A}^{C}\right)+c_{B}\left(m_{12} \widetilde{D}_{A B}^{C}-m_{22} \widetilde{D}_{A A}^{C}\right)}{c_{B} m_{12} \widetilde{D}_{A B}^{C}-c_{B} m_{22} \widetilde{D}_{A A}^{C}-c_{A} m_{12} \widetilde{D}_{B B}^{C}+c_{A} m_{22} \widetilde{D}_{B A}^{C}}
$$




$$
\frac{w_{C}}{w_{B}}=\frac{\left(1-c_{B}\right)\left(\left(1-c_{A}\right)\left(m_{22} \widetilde{D}_{B A}^{C}-m_{12} \widetilde{D}_{B B}^{C}\right)+c_{B}\left(m_{22} \widetilde{D}_{A A}^{C}-m_{12} \widetilde{D}_{A B}^{C}\right)\right)}{c_{B}\left(-\left(m_{22}\left(1-c_{A}\right)+m_{21}\right) \widetilde{D}_{21}^{C}+\left(\left(1-c_{A}\right) m_{12}+m_{11}\right) \widetilde{D}_{B B}^{C}+c_{B} m_{12} \widetilde{D}_{A B}^{C}-c_{B} m_{22} \widetilde{D}_{A A}^{C}\right)} ;
$$

where $\Delta_{m}=m_{11} m_{22}-m_{12} m_{21}, m_{\mathrm{ij}}=c_{\mathrm{j}}(\mathrm{kT})^{-1} \partial \mu_{\mathrm{j}} / \partial c_{\mathrm{i}}$ and $\mu_{1}$ is the chemical potential of component $A$ and $\mu_{2}$ is the chemical potential of component $B$.

Manning [29], amongst many others, has argued that the off-diagonal phenomenological coefficients should never be neglected since they play an important role by carrying diffusion correlation information. However, for the purposes of obtaining ratios of exchange frequencies, Equations 13-16 should provide rough estimates, but for greater accuracy it would be necessary to include the off-diagonal coefficients. The inclusion of the off-diagonal phenomenological coefficients then would require the use of the Manning or MAA diffusion kinetics formalisms from the very beginning and either formalism can be quite complicated to implement, involving the solution of a large set of non-linear equations. Details of the utilization of these formalisms in this context can be found in [30,31]. Perhaps surprisingly, in austenitic $\mathrm{Fe}-\mathrm{Ni}-\mathrm{Cr}$ ternary alloys, upon processing the interdiffusion data it was found that there was not in fact a great deal of difference in either the ratio of exchange frequencies or the resulting tracer correlation factors. There is presumably a subtle cancellation of errors. In other words, it would appear that Eqns. 13-16 can in fact be used directly (with caution) to obtain the ratios of the exchange frequencies with the results then being used as input to a Manning (or MAA) analysis in order to obtain the corresponding tracer correlation factors.
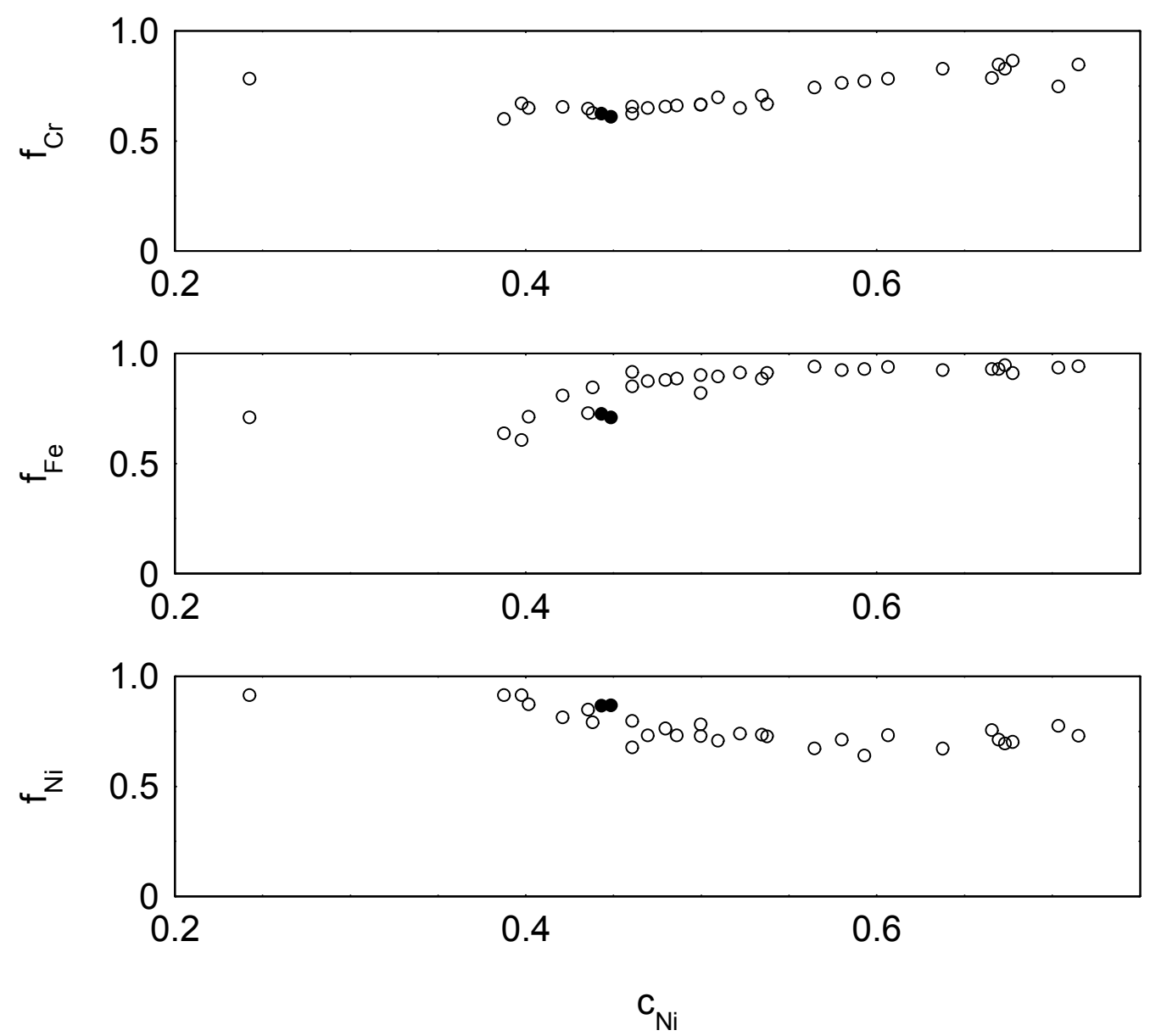

Fig. 4 Tracer correlation factors in austenitic $\mathrm{Fe}-\mathrm{Ni}-\mathrm{Cr}$ as functions of $\mathrm{Ni}$ and $\mathrm{Cr}$ composition at 1373K. Filled circles represent two points where all tracer diffusion coefficients are available; open circles - results of analysis of the interdiffusion and thermodynamic data by making use of the MAA approach. 
In Fig. 4 we give results for the tracer correlation factors from the full MAA analysis [30] of the extensive interdiffusion data provided by Duh and Dayananda [32] in the $\mathrm{Fe}-\mathrm{Ni}-\mathrm{Cr}$ system. In Fig. 4 we have also included the corresponding results for the analysis of the tracer diffusion data (for two points only) [33]. It can be seen that there is quite reasonable consistency between these two quite different types of diffusion measurements.

At high $\mathrm{Ni}$ compositions the $\mathrm{Fe}$ atoms show the least degree of correlation (highest correlation factors), whilst $N i$ atoms show the greatest degree of correlation (lowest correlation factors). The behavior of the $\mathrm{Cr}$ atoms falls between these extremes. On the other hand, at low $\mathrm{Ni}$ compositions the $\mathrm{Cr}$ atoms are the most correlated and $\mathrm{Ni}$ atoms are the least correlated whilst the behavior of $\mathrm{Fe}$ atoms falls in between. This crossing over of the correlation behavior appears to be a result of a change in the exchange frequencies with composition.

\section{Summary}

In this paper, we have discussed some of the theoretical procedures that have been established recently for analyzing intrinsic and interdiffusion data in binary and ternary alloy systems. Emphasis was put on extracting information to provide tracer correlation factors. Examples were taken from the intrinsic diffusion, interdiffusion and supporting tracer diffusion data when available in the $\mathrm{Cu}-\mathrm{Ni}, \mathrm{Ag}-\mathrm{Cd}, \mathrm{Ag}-\mathrm{Cd}-\mathrm{Zn}$ and $\mathrm{Fe}-\mathrm{Ni}-\mathrm{Cr}$ alloy systems.

\section{Acknowledgements}

We wish to acknowledge the support of this research by the Australian Research Council.

\section{References}

[1] A.D. Smigelskas and E.O. Kirkendall: Trans. AIME Vol. 171 (1947), p. 130

[2] N.L. Peterson in: Diffusion in Solids: Recent Developments, Eds. A.S. Nowick and J.J. Burton, Academic Press, New York 1975, p. 115

[3] J.N. Mundy in: Diffusion in Solids: Unsolved Problems, Ed. G.E. Murch, Trans Tech Publications, Zurich 1991, p. 1

[4] N.L. Peterson: J. Nucl. Mater. Vol. 69-70 (1978), p. 3

[5] A.D. Le Claire: J. Nucl. Mater. Vol. 69-70 (1978), p. 70

[6] G.E. Murch: Solid State Ionics Vol. 7 (1982), p. 177

[7] G.E. Murch: J. Phys. Chem. Solids Vol. 42 (1981), p. 227

[8] G.E. Murch and C.M. Bruff in: Diffusion in Solid Metals and Alloys, Ed. H. Mehrer, LandoltBörnstein New Series, Vol. 26, Springer-Verlag, Berlin, 1990, p. 279

[9] M.A. Dayananda in: Diffusion in Solid Metals and Alloys, edited by H. Mehrer, LandoltBörnstein New Series, Vol. 26, Springer-Verlag, Berlin, 1990, p. 372

[10] S.J. Rothman in: Diffusion in Crystalline Solids Eds. G.E. Murch and A.S. Nowick, Academic press, Orlando Florida 1984, p. 1

[11]I.V. Belova and G.E. Murch: Phil. Mag. Vol. 85 (2005), p. 1191

[12]J.R. Manning: Phys. Rev. B: Condens. Matter Vol. 4 (1971), p. 111

[13]G.E. Murch and I.V. Belova in: Mass and Charge Transport in Inorganic Materials II (Advances in Science and technology Vol. 37), Eds. P. Vincenzini and V. Buscaglia, Techna Faenza Italy 2003, p. 99

[14]L.K. Moleko and A.R. Allnatt: Phil. Mag. A Vol. 58 (1988), p. 677 
[15]A.R. Allnatt and A.B. Lidiard: Atomic Transport in Solids (Cambridge University Press 1993).

[16]L.S. Darken: Trans. Am. Inst. Min. (Metall.) Eng. Vol. 175 (1948), p. 184

[17]A.B. Lidiard: Acta Metall. Vol. 34 (1986), p. 1487

[18]L. Zhang, W.A. Oates, and G.E. Murch: Phil. Mag. B Vol. 60 (1989), p. 277

[19]I.V. Belova and G.E. Murch: Phil. Mag. A Vol. 80 (2000), p. 599

[20]I.V. Belova and G.E. Murch: Phil. Mag. A Vol. 80 (2000), p. 1469

[21]L.K. Moleko, A.R. Allnatt and E.L. Allnatt: Phil. Mag. A Vol. 59 (1989), p. 141

[22]I.V. Belova and G.E. Murch: Def. Dif. For. Vol. 224-225 (2004), p. 127

[23]I.V. Belova and G.E. Murch: Phil. Mag. Lett. Vol. 81 (2001), p. 661

[24]Y. Iijima, K. Hirano and M. Kikuchi: Trans. Jpn. Inst. Met. Vol. 23 (1982), p. 19.

[25]N.R. Iorio, M.A. Dayananda and R.E. Grace: Metall. Trans., Vol. 4 (1973), p. 1339

[26] A.B. Gardner, R.L. Sanders and R.L. Slifkin: Phys. Stat. Sol. Vol. 30 (1968), p. 96

[27]P.T. Carlson, M.A. Dayananda and R.E. Grace: Metall. Trans. Vol. 3 (1972), p. 819

[28]J.S. Kirkaldy and D.J. Young: Diffusion in the Condensed State (Inst. of Metals, London 1987).

[29]J.R. Manning: Metall. Trans. Vol. 1 (1970), p. 499

[30]I.V. Belova and G.E. Murch: Acta Mater. Vol. 50 (2002), p. 4617

[31]I.V. Belova, G.E. Murch, R. Filipek and M. Danielewski: Acta Mater. Vol. 53 (2005), p. 4613

[32] J.G. Duh and M.A. Dayananda: Dif. Def. Data, Vol. 39 (1985), p. 1

[33] S.J. Rothman, L.J. Nowicki and G.E. Murch: J. Phys. F: Met. Phys. Vol. 10 (1980), p. 383 
Diffusion and Thermodynamics of Materials

doi:10.4028/3-908451-35-3

Interdiffusion Data in Multicomponent Alloys as a Source of Quantitative Fundamental Diffusion Information

doi:10.4028/3-908451-35-3.1 